\section{Trabeculectomy in the UK: is there room for improvement?}

This issue of Eye sees the publication of the second report from the UK National Trabeculectomy Survey. ${ }^{1}$ The first paper $^{2}$ gave details of the patients in the study. The current paper describes the surgical methods and intraocular pressure outcomes 1 year following surgery.

The variations in operative techniques make interesting reading. The majority of surgeons in the UK use a superior rectus rather than corneal traction suture, do fornix-based rather than limbus-based conjunctival flaps, cut a rectangular rather than a triangular scleral flap and do not perform a paracentesis, use releasable sutures or any sort of mechanical punch. About one-fifth (22.6\%) of the consultants had a self-declared special interest in glaucoma ('glaucoma specialists'). These consultants differed from the majority in that they were more likely to use corneal traction sutures, triangular scleral flaps, releasable sutures and perioperative antimetabolites.

In view of the fact that $15 \%$ of the group had pre-operative pressures less than $21 \mathrm{mmHg}$, a modified success rate criterion of a final intraoperative pressure (IOP) less than twothirds of starting IOP off all medications was used. On this criterion $66 \%$ of the operations were successful. However, although the success rate in 'glaucoma specialists' was slightly higher $(69.6 \%$ vs $65.7 \%)$ this was not statistically significant. It is not possible to draw definitive conclusions from differences in technique in this study, as the level of use of some of these techniques was relatively low overall, even in those with a special interest in glaucoma. For instance, intraoperative antimetabolites (mostly intraoperative 5-fluorouracil) were used in only $14.7 \%$ of cases carried out by the 'glaucoma specialists'. This contrasts with the United States where a survey suggested that amongst ophthalmologists with an interest in glaucoma the use of intraoperative mitomycin in first-time trabeculectomy varied from $33 \%$ to $52 \% .^{3}$

The overall success rate using the more conventional criterion of an IOP less than $21 \mathrm{mmHg}$ of all medications in the survey was $84.2 \%$. This success rate is lower than that found in studies where surgery is performed in patients with virtually no prior medical treatment. These report success rates of $>90 \%$ at
1 year. ${ }^{4,5}$ However, as the study authors state, this group of patients accounts for less than 5\% of the survey population. Furthermore, the success rate in this national survey is considerably better than a recent multicentre survey across nine countries. This study found that more than $50 \%$ of patients were back on medication 1 year after surgery. ${ }^{6}$ In view of the comprehensiveness of this survey, the figures it provides will serve as a gold standard against which we can measure our personal and unit results, and look to improve in the future.

There are other important facts in this paper that should help us to improve our practice. Only $57.7 \%$ of the patients had a visual field performed in the year following surgery. We know that even with pressures less than $21 \mathrm{mmHg}$, visual field progression may continue, particularly if the pressures are not in the low teens. Therefore it is vital that further follow-up with field and disc assessment continues in patients even after apparently 'successful' surgery, as there is accumulating evidence that maintaining the IOP in the low teens better preserves visual function in both socalled high tension and normal tension glaucoma. 7,8

Finally, are there further ways that the results of surgery could be further improved in the UK? Small improvements of even $5-10 \%$ in the success rate may potentially affect the longterm prognosis for many thousands of patients per year in the UK if applied on a national scale. For instance in the Moorfields/Medical Research Council trial of intraoperative 5-fluorouracil (5-FU) versus placebo in first-time surgery, half the group have received intraoperative 5-FU. In the pressure range that is most likely to preserve vision $(15 \mathrm{mmHg}$ or less), ${ }^{7,8} 65 \%$ of all trial patients have pressures in this range versus $54.6 \%$ in the national survey. The trial patients receive active but simple bleb management in the post-operative period including posterior lip massage, releasable suture loosening and removal, and needling in the clinic. These procedures can be carried out in any centre. Pilot trials of newer more physiological antiscarring agents ${ }^{9}$ have also suggested that lower final IOPs may be possible in first-time surgery, and clinical trials of these promising agents are continuing.
P.T. Khaw

A.P. Wells

Moorfields Eye Hospital London EC1V 2PD, UK

P.T. Khaw, PhD, FRCP, FRCS, FRCOphth, FIBiol Professor of Glaucoma and Wound Healing \&

Consultant Ophthalmic Surgeon

Director, Wound Healing Research Unit

Glaucoma Unit and Department of Pathology Moorfields Eye Hospital and Institute of Ophthalmology Bath Street

London EC1V 9EL, UK

Tel/fax: +44 (0)207608 6887

e-mail: p.khaw@ucl.ac.uk

This work has been supported in part by MRC G9330070. This work was undertaken by the authors who received a proportion of funding from the NHS executive. The views expressed in this publication are those of the authors and not necessarily of the NHS executive 
In conclusion, this landmark survey has provided us with the national standards for trabeculectomy in the United Kingdom. We should all read the figures carefully, examine our own results and use this survey as a catalyst to see whether we can further improve the surgical management of all our patients undergoing glaucoma surgery.

\section{References}

1. Edmunds B, Thompson JR, Salmon JF, Wormald RP. The National Trabeculectomy Survey. II. Variations in operative technique and outcome. Eye 2001;15:441-8.

2. Edmunds B, Wormald RP, Thompson JR, Salmon JF. The National Trabeculectomy Survey. I. Sample and methods. Eye 1999;13:524-30.

3. Chen PP, Yamamoto T, Sawada A, Parrish RK, Kitazawa Y. Use of antifibrosis agents and glaucoma drainage devices in the American and Japanese glaucoma societies. J Glaucoma 1997;6:192-6.
4. Migdal C, Gregory W, Hitchings RA. Long term functional outcome after early surgery compared with laser and medicine in open angle glaucoma. Ophthalmology 1994;101:1651-7.

5. Jay JL, Allan D. The benefit of early trabeculectomy versus conventional management in primary open angle glaucoma relative to severity of disease. Eye 1989;3:528-35.

6. Hitchings RA. Efficacy of glaucoma treatment. In: Jonsson Krieglestein GK, editors. ISIS Medical Media 1998;153-61.

7. Collaborative normal-tension glaucoma study group. Comparison of glaucomatous progression between untreate patients with normal-tension glaucoma and patients with therapeutically reduced intraocular pressures. Am J Ophthalmol 1998;126:487-97.

8. AGIS Investigators. The Advanced Glaucoma Intervention study (AGIS). 7. The relationship between control of intraocular pressure and visual field deterioration. Am J Ophthalmol 2000;130:429-40.

9. Siriwardena D, Khaw PT, King AJ, Donaldson ML, Migdal C Cordeiro MF. A randomised double-masked placebocontrolled trial of human anti-TGFbeta2 monoclonal antibody (CAT-152): a potential new modulator of wound healing following trabeculectomy. Ophthalmology 2001; in press. 